In this research, a numerical investigation has been conducted to analyze the dynamic load of the composite structure. The composite structure was solved and analyzed using the static structure tool. There is a physical model that has been imported and meshes have been done accordingly. Shear stress, von-mises stress, and total deformation are all considered as part of the analysis. Using finite elements to simulate the composite structure and response to the applied loads. Because dynamic loads were regularly applied, frequency response was examined. In this study after verification of this computer simulation with experimental results and the results showed it was confidence $95 \%$. This percentage confidence allows proceeding numerical analysis. Deformation of the entire structure has been computed and found to be $10 \mathrm{~mm}$, which is the maximum amount of overall deformation that can be caused by the applied load, In the Y-axis, a dynamic load was applied. The imposed dynamic load has been studied numerically and interpreted in terms of shear stresses. As a result of the maximum applied load, the maximum shear stress is $10 \mathrm{MPa}$. The complete composite construction was subjected to Von-Mises stress measurements. The structure's ability to withstand these stresses was determined by conducting a series of tests. The greatest von mises stress that can be applied in this study was $40 \mathrm{MPa}$. An investigation of how to react to vibration has already been carried out. In the numerical results, it was found that the reaction to the vibration was inconsistent. In terms of amplitude, the highest values may be found at $200 \mathrm{~Hz}$, while the lowest values can be found at $20 \mathrm{~Hz}$

Keywords: ANSYS, FEM, Fractional corrosion, L-shape sandwich, USV, Vibration, Von-mises stresses, shear stresses, total deformation, vibration response
UDC 531

DOI: $10.15587 / 1729-4061.2022 .253545$

\title{
IMPLEMENTATION OF THE NUMERICAL ANALYSIS OF DYNAMIC LOADS ON THE COMPOSITE STRUCTURE EMPLOYING THE FE METHOD
}

\author{
Kussay Ahmed Subhi \\ Correspondingauthor \\ Assistant Professor* \\ E-mail: kussaysubhi@atu.edu.iq \\ Emad Kamil Hus ein \\ Assistant Professor*
}

Shaymaa Abdul Khader Al-Jumaili Assistant Professor*

Zaid Ali Abbas

Researcher

Department of Electrical and Electronics Engineering

Universiti Putra Malaysia

Serdang, Selangor, Malaysia, 43400

*AI-Mussaib Technical College TCM

Al-Furat Al-Awsat Technical University ATU Al-Mussaib, Babil, Iraq, P.O. Box 51006

Received date 10.012022 Accepted date 21.02.2022 Published date 28.02.2022
How to Cite: Subhi, K. A., Hussein, E. K., Al-Jumaili, S. A. K., Abbas, Z. A. (2022). Implementation of the numerical analysis of dynamic loads on the composite structure employing the FE method. Eastern-European Journal of Enterprise Technologies, 1 (7 (115)), 42-47. doi: https://doi.org/10.15587/1729-4061.2022.253545

\section{Introduction}

Static structures are widely used in ultra-precision machining, optical focusing, micro/nanomechanical testing, and micromanipulation because of their compact construction, non-electromagnetic interference, fast reaction, and high positioning resolution [1]. It is usual for stepping piezoelectric actuators to be built to meet the needs of applications where both high positioning resolution and large working stroke are required [2]. Stepping piezoelectric actuators can be divided into ultrasonic driving type, inchworm driving type, stickslip driving type, parasitic motion driving type, and so on. The stick-slip piezoelectric actuators, which have a simple structure and control, have been widely used. In order to further improve the performance of stick-slip piezoelectric actuators, numerous studies have been conducted in recent years. A stick-slip placement stage, for example, was proposed to improve loading capacity by separating the driving and moving units [3].
In order to improve the stability of the motion, Liu and Chu used the gravity of the rotor or slider to maintain a consistent contact status. [3] came up with the notion of creating a forward frictional force to counteract the reversed frictional force in stick-slip piezoelectric actuators. Improving the driving frequency of stick-slip piezoelectric actuators is a standard approach for increasing motion speed. For instance, stick-slip's piezoelectric actuator reached a maximum motion speed of $6.057 \mathrm{~mm} / \mathrm{s}$ at a driving frequency of $5000 \mathrm{~Hz}$ by creating a Z-shaped flexure hinge [4]. To further increase the speed of their stick-slip piezoelectric actuator, they used a parallelogram-type flexure hinge [5]. This piezoelectric actuator was further improved upon utilizing a triangular compliant drive mechanism, and it obtained a remarkable speed of $46.67 \mathrm{~mm} / \mathrm{s}$ at 3500 cycles per second [6]. It is possible for these actuators to move at a relatively fast speed, but their operating frequencies are also fairly high at more than $2000 \mathrm{~Hz}$. The stability of the system is compromised and the stick-slip actuator experiences increased wear and tear while operating at a high frequency [7]. 
There was a tendency to position actuators on the amplifier's exterior, resulting in bulky structures. Because of its small size, the bridge-type amplifier (BTA) has been utilized in compliant positioning systems. BTA's dynamic performance is also inferior due to the fact that PEA restricts its size and shape. The performance of micro/nano positioners can be improved by developing a novel compliant amplification mechanism with a small size and good dynamic characteristics. When designing micro/nano positioners, keep in mind the importance of motion coupling. To achieve input and output decoupling, many motion decoupling techniques have recently been developed indeterminate symmetric construction to accomplish varied decoupling of axial motion [8]. As much cross-coupling as possible was achieved using a decoupled XYZ. Flexure Roberts mechanism was used in the parallel-kinematic positioner devised [9]. This positioner has a cross-coupling limit of $1.7 \%$. Asymmetric stiffness mechanism motion coupling has been reduced for most of the positioners mentioned above using overconstrained structures [10]. However, few studies have focused on motion coupling caused by inconsistent central axes of the PEA and input mechanism, which will have a major impact on positioner motion coupling.

The effects of strain rate on hysteresis loops and fatigue life were examined at room temperature. In order to describe the isothermal tiredness of the material, they used the notion that damage accumulates in solder material and applied it to the theory of damage mechanics to arrive at a definition of fatigue failure. Experiments have proven that the model is accurate in predicting how fatigue and cyclical behavior will impact load rates. The effects of temperature and microstructure on bereavement of harm dynamics were required to be fully understood. Thermal-aging copper/lead-free soil joints that have been as-soldered are monotonous and cyclically distorted.

Shock-induced failure of solder junctions owing to ultrasonic vibration welding can now be tested using an improved simulation, according to [11]. The dynamic vibration process was investigated using FEM during the welding process, according to the findings of the study. A number of researchers, including (have stated that FE approaches have been developed to anticipate the damage produced by ultrasonic welding in the plastic components. Many applications call for numerical models of thin metal with ultrasonic vibration. To show ultrasonic welding in a FEM, the mechanical properties of the top foil and substrate are very important [12].

Therefore, studies that are devoted that the elements can be simulated using a finite-element model, but still confront major geometry issues. Therefore, Research will focus on creating composite material for mechanical features and analyzing superior property values through efficient finite element analysis.

\section{Literature review and problem statement}

Primary structurally loaded components of commercial and military aeronautical vehicles have long made use of composite materials. Composite materials, on the other hand, are susceptible to multiple types of damage throughout the production, transportation, and operating processes. Low-velocity impact damages such as fiber breakage, matrix cracks, and delaminations, which are difficult to detect and have the potential to seriously compromise the structural integrity of composite structures, are a major problem in the composite structure design. Structure health monitoring (SHM) is becoming increasingly popular because of recent innovations in sensing technologies, as well as advancements in processing and communication [13].

Composite structure impact loads are typically identified by determining the impact position and then recreating the impact force history. There have been a lot of studies done and reported on impact load identification during the past few years [14]. It is possible to locate the impact site by employing joint time-frequency analysis to evaluate the impact-induced stress waves. The impact location can be determined by solving a series of nonlinear equations explaining the relationships between impact location, group velocities, and times-of-flight (TOFs). However, this method can only be used to estimate the impact site without providing any information regarding the impact force. Impact forces can be reconstructed using analytical methods described [15] which find relationships between impact force and reactions with known impact site in isotropic plates. To find the inverse impact force history, use this connection. Analytically, it's difficult to establish an accurate correlation between composite and complicated structures. The use of intelligent methods is also applied to identify the impact. It has been demonstrated that neural networks may be used to identify the impact site and degree of impact force concurrently [16]. However, it requires an exhausted impact data set through training tests, which may not be practicable for practical usage. There have only been methods based on system optimization theory that have been successful in determining the impact site and impact force history [17]. This robust impact identification. For laminated composite plates and stiffened composite panels, this method to identify the impact load.

In this study, researchers have found a small finite element model regime to investigate element failure, but they still face significant structure geometry challenges. As a result, the focus of this research will be on developing solder junction mechanical characteristics and investigating superior property properties through efficient FE analysis.

\section{The aim and objectives of the study}

The main aim of the study is to investigate the effect of the dynamic load on the composite structure numerically. This development will increase the mechanical performance composite of the L-shape structure.

To achieve this aim, the following objectives are accomplished:

- to investigate the response to the applied load has been investigated and explained clearly;

- to calculate von Mises stresses have been undertaken for the entire structure due to the applied load;

- to determine shear stresses due to the applied load were conducted and analyzed basely;

- to determine total deformation due to the reputation of the applied loads has been investigated and analyzed accordingly. In this study, the effect on the entire structure has been considered.

\section{Materials and methods}

In this research to carry out the simulation process, there are several steps must be followed accordingly. The first step is to set up the finite element to be suitable for use. Secondly, import geometry and mesh it basely. To run the simulation 
process, boundary conditions must be defined in terms of material properties.

\section{1. Finite Element Analysis set up}

Static, Modal, and Transitional Finite Element Analysis was used in this study. Modal analysis is used to identify the equipment's normal frequencies and model forms. Feature parameters such as natural frequencies and design shapes are critical. In the event of a dynamic loading situation. Alloy structural stability is determined by resonance frequencies and fashion shapes. If the frequency expected by the model is similar to or at a resonant frequency, the computer will undergo unregulated non-linear deformation, which can lead to a system failure or inaccurate test results.

\section{2. Geometry and Meshing}

An l-shaped structure of composite materials was used in the study. For the purposes of creating the project's geometry, AUTOCAD software was utilized. In order to produce the mesh, we used ANSYS mechanical, which was informed by our research into [18]. Pave meshes of three and four types were used in the more sophisticated models, while quad meshes were used in the rest of the models. ANSYS, as illustrated in Fig. 1, provides a finished mesh as displayed.

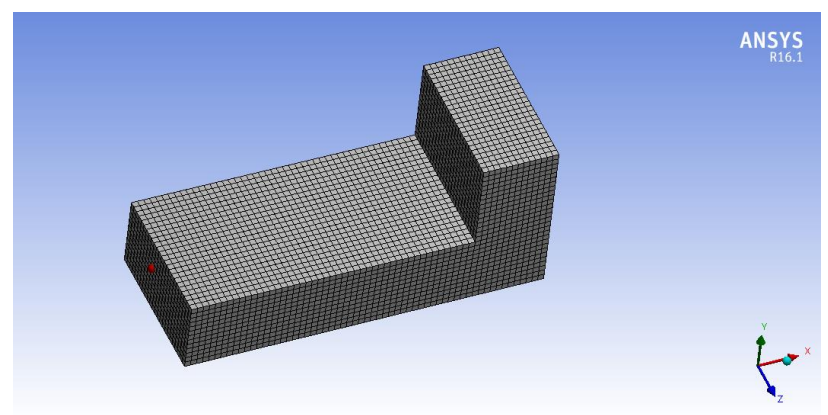

Fig. 1 The meshing model

Mesh has been created using a static structure tool in the ANSYS. it has been done with a soft option with a suitable number of elements. Elements have been distributed equally to ensure quality in the outcome results.

\section{3. Boundary Conditions}

In this research, the L-Shape sandwich structure was carried out to investigate the characters of this alloy within a particular period due to high frequencies. The following table shows the structure features as shown in Table 1.

Table 1

Mechanical properties of L-Shape sandwich structure

\begin{tabular}{|c|c|c|c|}
\hline $\begin{array}{c}\text { L-Shape } \\
\text { materials }\end{array}$ & Poisson's ratio & Density & $\begin{array}{c}\text { Young's Modulus of } \\
\text { elasticity }\end{array}$ \\
\hline Units & $\mathrm{gm} / \mathrm{cm}^{3}$ & $\mathrm{gm} / \mathrm{cm}^{3}$ & $\mathrm{GPA}$ \\
\hline Values & 0.3 & 5.4 & 12.1 \\
\hline
\end{tabular}

In order to carry out the results, three main elements should be provided. Poisson's ratio, Density, and Young's Modulus of elasticity these elements are defining the material inside the ANSYS library.

\section{Results of Numerical analysis of L-shape composite structure}

\section{1. Grid independent explanation of numerical analy-} sis of the composite structure

ANSYS 16.1's vibration response tool was used to do preprocessing on the structure. In this study, a local mesh model was generated. Linear loads are sufficient for the current type of elements. As illustrated, seven methods were tested with the same applied load of $1000 \mathrm{~N}$ and the same overall deflection. Based on the findings of the simulations that were conducted. The curve becomes horizontal with 9000 components at a total deflection of $0.22 \mathrm{~mm}$. That the meshed model is convergent has been demonstrated in this experiment.

\section{2. Validation of the previous study of the composite} structure

Validation must be performed under the same settings as the experimental work in order to continue using the existing boundary conditions. Fig. 2 shows the results of a simulation method that was used. Fig. 2 shows the relationship between the experimental simulation and its effects. The current results have been compared with studies accordingly [19]. After comparing the results of the computer simulation with those of the experiments, the researchers came to a $93 \%$ conclusion.

The results have proven that it is valid to proceed with the new attempts. This step was considered is the most important one among other steps. Once, the first results of the simulation are varied other results will be confident accordingly.

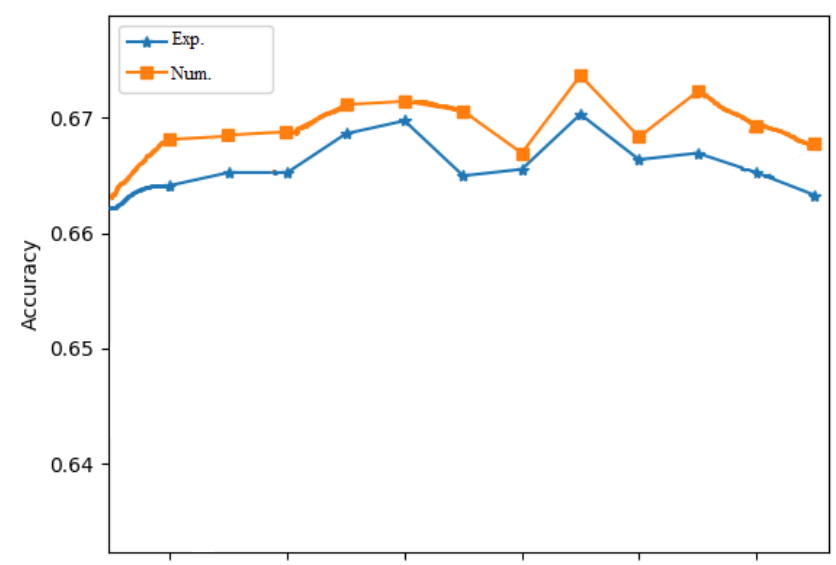

Fig. 2. Comparison between simulation results and experiment results

5. 3. Response to the vibration of the L-shape of the composite structure

The L-Shape structure response to vibration in terms of amplitude (stress) varies, according to the numerical data. An ANSYS tool called Harmonic Response was used to do this research. Amplitude (stress) was used as the primary metric to assess the structural vibration response. As can be seen in Fig. 3, the amplitude has been reduced to its smallest possible value. At $100 \mathrm{~Hz}$, the amplitude reached its highest point. The purpose of this test is to get insight into the mechanical response of materials to the frequencies being applied. As a result, the vibration response is unstable for the material employed.

In this study, the dynamic load was applied raptly and Fig. 3 showed the response of the composite structure to the applied load accordingly. Where the maximum responses have been done two times during the simulation process. 


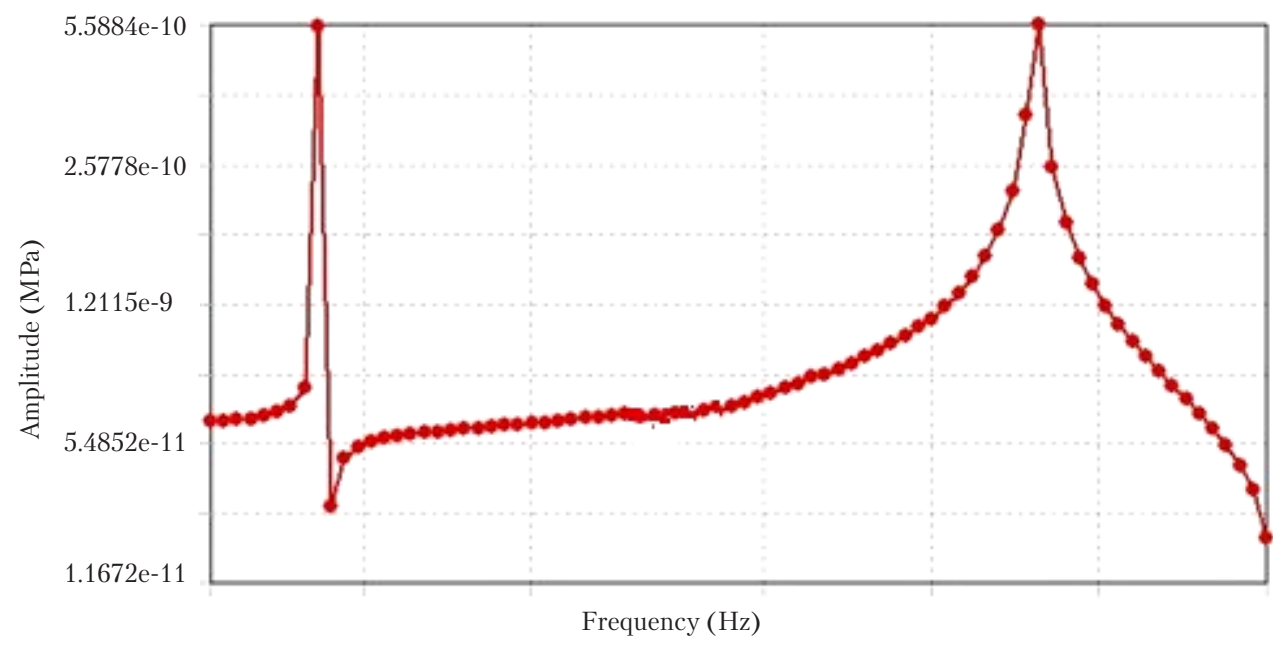

Fig. 3. Vibration response in terms of Amplitude ( $\mathrm{MPa}$ )

5. 4. Results of Numerical analysis due to applied dynamic loads

To assess the shear strength of an 1-shape structure this research used high frequencies and a precise flow period.

\section{4. 1. Implementation of shear stresses}

Due to the precise time range of flow, ANSYS software has been used to simulate the shear strength l-shape sandwich. As shown in Fig. 4, the FE model has yielded this outcome.

The $10 \mathrm{kHz}$ frequency was used in this trial with a flow time interval of six $(0,1.5,3,4.5,6 \mathrm{sec})$. At $3 \mathrm{sec}$, the max performance (stress) was $10 \mathrm{MPa}$. As well as the results, it was shown that the lowest output occurred at the highest flow time as flow $8.1 \mathrm{MPa}$ at 6 sec. Fig. 5 indicates the frequency implementation for a different time span as shown in Fig. 5.

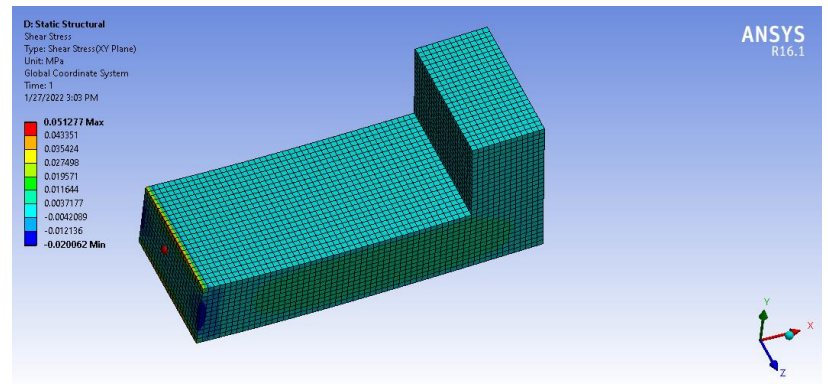

Fig. 4. Shear stress due to dynamic loads

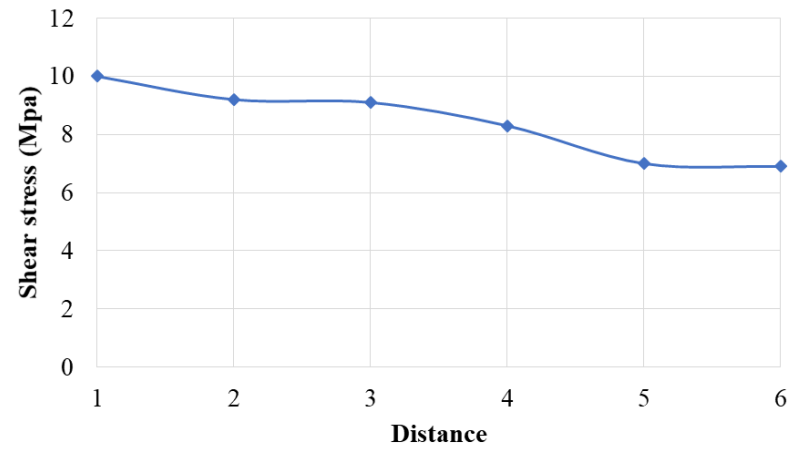

Fig. 5. Shear stress due to dynamic load

Shear stresses have been collected through different distances during the applied loads. Where the simulation results stated the maximum results have occurred when the distance is 0.9 and the minimum value of shear stress reaches the minimum value at 6 .

\section{4. 2. Implementation of von-mises stress}

Fig. 6 shows the graphical effect of von-mises stress due to applied dynamic loads L-shape sandwich structure. Where the two substances are subjected to horizontal (Y direction) forces that lead to shear stress. The force has been applied with an organized range of frequency. This investigation was carried out by applying these loads.

The numerical results stated that a $120 \mathrm{kHz}$ frequency and a flow time interval of six minutes were used in this experiment $(0,1.5,3,4.5,6 \mathrm{sec})$. The maximum force (performance) was $40 \mathrm{MPa}$ at 3 seconds. Additionally, the lowest production was found to occur at a flow rate of $33 \mathrm{MPa}$ for 6 seconds. For a different time span, the dynamic load's implementation is depicted in Fig. 7.

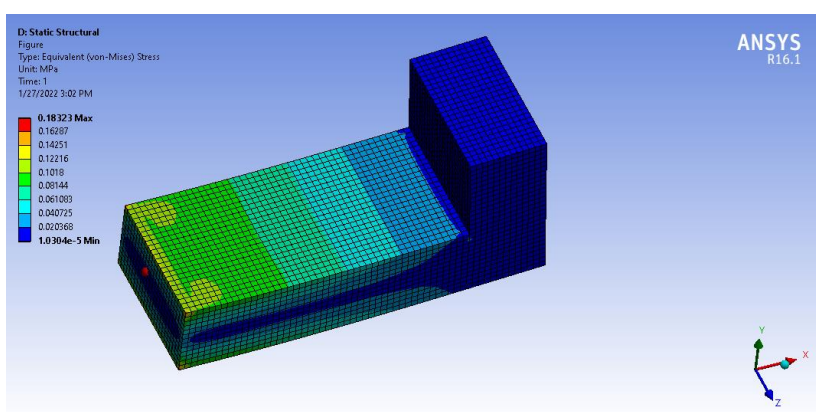

Fig. 6. The graphical effect of Von-bises stress on L-SAHPE structure due to dynamic loads

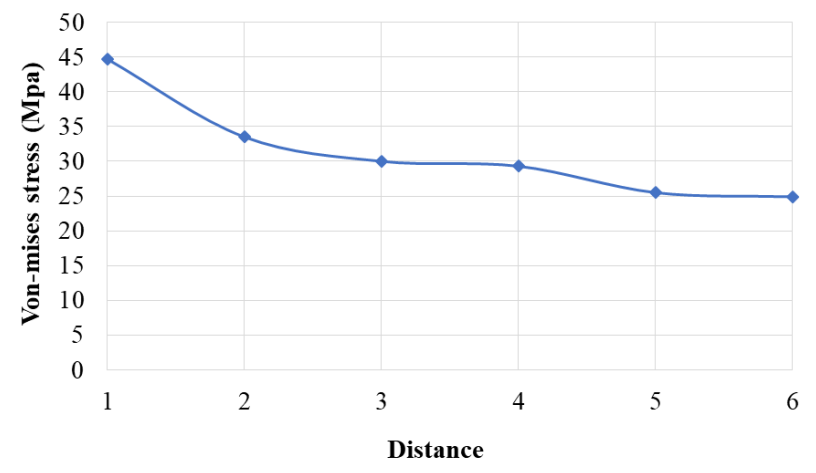

Fig 7. Von-mises stress due to dynamic loads 
This is seen in Fig. 7, which shows the simulation results. The graph demonstrates that the two curves are connected by two frequencies, $120 \mathrm{~Hz}$ and $100 \mathrm{~Hz}$. There are stronger intensity values attributed to the simulation than to the findings produced by $20 \mathrm{kHz}$ at the same interval.

\section{4. 3. Total deformation calculations}

Total defamatory data acquired by ANSYS Software is depicted graphically in Fig. 8. The entire simulation was carried out using a static structure tool. Fig. 8 depicts the deformation of an L-shaped sandwich structure as a result of dynamic stresses. Shear stress occurs when two materials are subjected to horizontal $(Y)$ forces that cause them to be displaced. The force has been administered systematically, using a set of predetermined frequencies. These loads were used to conduct this experiment.

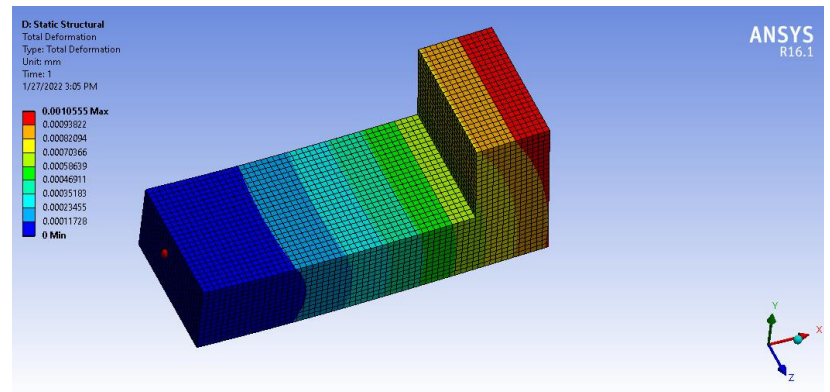

Fig. 8. Ehe effect of total deformation on the structure

Fig. 8 showed the effect of the total deformation due to applied load graphically. Where the results have shown that the maximum deformation is $10 \mathrm{~mm}$ and occurred at the end of the body and the minimum deformation occurred on the front end of the body.

\section{Discussion of the results of the numerical analysis of L-shape composite structure}

In this study, three main parameters have been considered to be investigated and analyzed. The first of the main parameters is shear stress due to the dynamic load on the composite structure. The result has explained the effect of the loads on the L-Shape structure in terms of shear stresses. This process has been shown in Fig. 4 where all body has been subjected to the dynamics loads and the simulation process shows the responses of the body to the loads in terms of the shear stresses. The results can be explained in calculations shear stresses value with each step of the applied load as shown in Fig. 5.
The second parameter that has been undertaken is total deformation to all structures within the applied dynamic load as shown in Fig. 8. Thirdly, von mises stress is also employed to be investigated for the applied load. The results can be explained in calculations of deformation in each step of the load at different distances.

The feature of the current method is to evaluate the main input in the Ansys software and solve the inputs basely. The finite element method has proven its effectiveness in the previous studies for that purpose it has been employed in this study. Composite of L-shape structure was created, modeled, and meshed using static structure tool in Ansys. This model was defined using the ANSYS library. Dynamic loads were applied and investigated. Thus, the numerical results that were got from the numerical analysis based on the FE method have been compared with the experiment results accordingly as shown in Fig. 4. The confidence of the numerical result with previous results will prove that the simulation process setup is made accordingly.

The limitation of current research is with dynamic loads. With frequency (limited). The output is limited on the shear stress, total deformation, and von mises stress.

This result can be further modified in the future using different methods in solving input and different software. The frequency range can be extended more with higher or lower. Materials also can be developed or changed.

Several challenges have been faced in numerical analysis, the most effective one is setting mesh with a suitable number of elements.

\section{Conclusions}

1. Response to the vibration has already been conducted to be investigated accordingly. The numerical results revealed that the response to the vibration fluctuated. The maximum amplitude occurs at a frequency of $200 \mathrm{~Hz}$ and the minimum amplitude reached $20 \mathrm{~Hz}$.

2. Shear stresses have been analyzed and explained in the numerical analysis to the applied dynamic load. the maximum shear stress load to the applied load is $10 \mathrm{MPa}$ this with the maximum applied value of the load.

3 . Von-mises stresses were determined, of the entire body of the composite structure. These stress were investigated to indicate the capacity of the structure. In this study, the numerical results stated that the maximum von mises stress can be used is $40 \mathrm{MPa}$.

4. Total deformation, of the entire structure, has been calculated and the results stated that the maximum value of total deformation due to the applied load is $10 \mathrm{~mm}$. dynamic load was applied in Y-axis.

\section{References}

1. Abbas, E. N., Jweeg, M. J., Al-Waily, M. (2018). Analytical and numerical investigations for dynamic response of composite plates under various dynamic loading with the influence of carbon multi-wall tube nano materials. International Journal of Mechanical \& Mechatronics Engineering IJMME-IJENS, 18 (06), 1-10. Available at: http://ijens.org/Vol_18_I_06/180506-9292-IJMME-IJENS.pdf

2. Dey, S., Mukhopadhyay, T., Sahu, S. K., Adhikari, S. (2018). Stochastic dynamic stability analysis of composite curved panels subjected to non-uniform partial edge loading. European Journal of Mechanics - A/Solids, 67, 108-122. doi: https://doi.org/10.1016/ j.euromechsol.2017.09.005

3. Abedini, M., Zhang, C. (2021). Dynamic performance of concrete columns retrofitted with FRP using segment pressure technique. Composite Structures, 260, 113473.doi: https://doi.org/10.1016/j.compstruct.2020.113473 
4. Garg, A., Chalak, H. D., Belarbi, M.-O., Zenkour, A. M., Sahoo, R. (2021). Estimation of carbon nanotubes and their applications as reinforcing composite materials - an engineering review. Composite Structures, 272, 114234.doi: https://doi.org/10.1016/ j.compstruct.2021.114234

5. Itu, C., Öchsner, A., Vlase, S., Marin, M. I. (2018). Improved rigidity of composite circular plates through radial ribs. Proceedings of the Institution of Mechanical Engineers, Part L: Journal of Materials: Design and Applications, 233 (8), 1585-1593. doi: https:// doi.org/10.1177/1464420718768049

6. Tarfaoui, M., Nachtane, M., Khadimallah, H., Saifaoui, D. (2017). Simulation of Mechanical Behavior and Damage of a Large Composite Wind Turbine Blade under Critical Loads. Applied Composite Materials, 25 (2), 237-254. doi: https://doi.org/10.1007/ s10443-017-9612-x

7. Garoz, D., Gilabert, F. A., Sevenois, R. D. B., Spronk, S. W. F., Van Paepegem, W. (2019).Consistent application of periodic boundary conditions in implicit and explicit finite element simulations of damage in composites. Composites Part B: Engineering, 168, 254-266. doi: https://doi.org/10.1016/j.compositesb.2018.12.023

8. Madenci, E., Özkılıç, Y. O., Gemi, L. (2020). Experimental and theoretical investigation on flexure performance of pultruded GFRP composite beams with damage analyses.Composite Structures, 242, 112162.doi: https://doi.org/10.1016/j.compstruct.2020.112162

9. Tarfaoui, M., Shah, O. R., Nachtane, M. (2019). Design and Optimization of Composite Offshore Wind Turbine Blades.Journal of Energy Resources Technology, 141 (5).doi: https://doi.org/10.1115/1.4042414

10. Munian, R. K., Mahapatra, D. R., Gopalakrishnan, S. (2018). Lamb wave interaction with composite delamination. Composite Structures, 206, 484-498. doi: https://doi.org/10.1016/j.compstruct.2018.08.072

11. Subhi, K. A., Tudor, A., Hussein, E. K., Wahad, H., Chisiu, G. (2018). Ex-Vivo Cow Skin Viscoelastic Effect for Tribological Aspects in Endoprosthesis. IOP Conference Series: Materials Science and Engineering, 295, 012018. doi: https://doi.org/10.1088/ $1757-899 x / 295 / 1 / 012018$

12. Hussein, E. K., Subhi, K. A., Gaaz, T. S. (2021). Effect of Stick - Slip Phenomena between Human Skin and UHMW Polyethylene. Pertanika Journal of Science and Technology, 29 (3).doi: https://doi.org/10.47836/pjst.29.3.06

13. Subhi, K. A., Tudor, A., Hussein, E. K., Wahad, H. S. (2017). The adhesion and hysteresis effect in friction skin with artificial materials. IOP Conference Series: Materials Science and Engineering, 174, 012018. doi: https://doi.org/10.1088/1757-899x/174/1/012018

14. Patel, S., Vusa, V. R., GuedesSoares, C. (2019). Crashworthiness analysis of polymer composites under axial and oblique impact loading. International Journal of Mechanical Sciences, 156, 221-234. doi: https://doi.org/10.1016/j.ijmecsci.2019.03.038

15. Zhou, J., Guan, Z., Cantwell, W. J. (2018). The energy-absorbing behaviour of composite tube-reinforced foams. Composites Part B: Engineering, 139, 227-237. doi: https://doi.org/10.1016/j.compositesb.2017.11.066

16. Guzman-Maldonado, E., Wang, P., Hamila, N., Boisse, P. (2019). Experimental and numerical analysis of wrinkling during forming of multi-layered textile composites. Composite Structures, 208, 213-223. doi: https://doi.org/10.1016/j.compstruct.2018.10.018

17. Bedon, C., Fragiacomo, M. (2019). Numerical analysis of timber-to-timber joints and composite beams with inclined self-tapping screws. Composite Structures, 207, 13-28. doi: https://doi.org/10.1016/j.compstruct.2018.09.008

18. Han, S., Meng, Q., Araby, S., Liu, T., Demiral, M. (2019). Mechanical and electrical properties of graphene and carbon nanotube reinforced epoxy adhesives: Experimental and numerical analysis. Composites Part A: Applied Science and Manufacturing, 120, 116-126. doi: https://doi.org/10.1016/j.compositesa.2019.02.027

19. Wang, K., Inman, D. J., Farrar, C. R. (2005). Modeling and analysis of a cracked composite cantilever beam vibrating in coupled bending and torsion. Journal of sound and vibration, 284 (1-2), 23-49. Available at: https://www.infona.pl/resource/bwmeta1. element.elsevier-116d13c3-5285-311c-a9b2-080f8f7c0a3d 